\title{
Fatores de risco para o desmame precoce no período neonatal: uma revisão integrativa da literatura
}

Risk factors for early weaning in the neonatal period: an integrative literature review

Factores de riesgo de destete temprano en el periodo neonatal: revisión integrativa de la literatura

\begin{abstract}
RESUMO
Introdução: o leite materno é o alimento ideal para criança no período neonatal, por possuir todos os nutrientes necessários para seu crescimento e desenvolvimento saudável. Apesar desses benefícios, as prevalências de Aleitamento Materno Exclusivo estão abaixo das recomendações do Ministério da Saúde. Objetivo: analisar os fatores de risco para o desmame precoce no período neonatal destacados na literatura nacional e internacional. Método: tratou-se de uma revisão integrativa de literatura indexada nas bases de dados PUBMED, CINAHL, LILACS/BDENF/IBECS via BVS, WEB of SCIENCE e SCOPUS. A amostra constituiu-se de 12 artigos publicados entre 2001 e 2019. Resultados: identificou-se fatores de risco psicológicos, anatômofisiológicos ou patológicos; introdução de fórmula artificial/suplementação alimentar; relacionados à técnica de amamentação; uso de utensílios culturais como chupetas e mamadeiras; retorno às atividades laborais e/ou escolares. Conclusão: a análise dos artigos evidenciou a complexidade da prática do aleitamento materno e o caráter multifatorial do desmame precoce.
\end{abstract}

DESCRITORES: Aleitamento Materno; Fatores de risco; Desmame; Recém-nascido.

\section{ABSTRACT}

Background: breast milk is the ideal food for children in the neonatal period, as it has all the necessary nutrients for their growth and healthy development. Despite these benefits, the prevalence of exclusive breastfeeding is below the recommendations of the Ministry of Health. Objective: to analyze the risk factors for early weaning in the neonatal period highlighted in national and international literature. Method: it was an integrative literature review indexed from the PUBMED, CINAHL, LILACS/BDENF/IBECS databases by VHL, WEB of SCIENCE and SCOPUS. The sample were 12 full papers published between 2001 and 2019. Results: psychological, anatomical and pathological risk factors were identified; introduction of artificial recipe/food supplementation; regarding to the breastfeeding technique; use of common tools such as pacifiers and bottles; return to employment and/or school activities. Conclusion: the analysis of the full papers showed the complexity of breastfeeding and the multifactorial nature of early weaning. DESCRIPTORS: Breast Feeding; Risk Factors; Weaning; Infant, Newborn.

\section{RESUMEN}

Introducción: la leche materna es el alimento ideal para los niños en el período neonatal, ya que posee todos los nutrientes necesarios para su crecimiento y desarrollo saludable. A pesar de estos beneficios, la prevalencia de la lactancia materna exclusiva está por debajo de las recomendaciones del Ministerio de Salud. Objetivo: analizar los factores de riesgo para el destete precoz en el período neonatal destacados en la literatura nacional e internacional. Método: se trató de una revisión de integradora de literatura indexada en las bases de datos PUBMED, CINAHL, LILACS/BDENF/ IBECS vía BVS, WEB of SCIENCE y SCOPUS. La muestra estuvo compuesta por 12 artículos publicados entre 2001 y 2019. Resultados: se identificaron factores de riesgo psicológicos, anatómicos y patológicos; introducción de fórmulas artificiales / complementos alimenticios; relacionado con la técnica de lactancia materna; uso de herramientas culturales como chupetes y biberones; regreso al trabajo y / o actividades escolares. Conclusión: el análisis de los artículos mostró la complejidad de la lactancia materna y la naturaleza multifactorial del destete precoz.

DESCRIPTORES: Lactancia Materna; Factores de Riesgo; Destete; Recién Nacido.

RECEBIDO EM: 17/08/2020 APROVADO EM: 31/08/2020

\section{Itamara Duarte do Lago}

Discente do curso de enfermagem - Campus Amilcar Ferreira Sobral da Universidade Federal do Piauí (UFPI), Floriano, Piauí. ORCID: 0000-0001-6192-7262 


\section{Mariana Mesquita Silva}

Enfermeira - Campus Amilcar Ferreira Sobral da Universidade Federal do Piauí (UFPI), Floriano, Piauí.

ORCID: 0000-0001-9126-552X

\section{Meirylene dos Santos Ferreira}

Enfermeira. Especialista em Saúde da Família. Coordenadora de Educação Permanente em Saúde do Hospital Regional Tibério Nunes (HRTN), Floriano, Piauí.

ORCID: 0000-0002-0422-2984

\section{Karla Nayalle Souza Rocha}

Enfermeira. Mestre em Enfermagem pelo Programa de Pós-graduação em Enfermagem da Universidade Federal do Piauí (UFPI). Docente do Curso Técnico em Enfermagem do Colégio Técnico de Bom Jesus - Campus Cinobelina Elvas da Universidade Federal do Piauí (UFPI), Bom Jesus, Piauí.

ORCID: 0000-0001-7184-003X

\section{Ruth Cardoso Rocha}

Enfermeira. Mestre em Enfermagem pelo Programa de Pós-graduação em Enfermagem da Universidade Federal do Piauí (UFPI). Docente do Curso de Bacharelado em Enfermagem - Campus Amilcar Ferreira Sobral da Universidade Federal do Piauí (UFPI), Floriano, Piauí.

ORCID: 0000-0001-6702-6844

\section{Maria Augusta Rocha Bezerra}

Enfermeira. Doutora em Enfermagem pelo Programa de Pós-graduação em Enfermagem da Universidade Federal do Piauí (UFPI). Docente do Curso de Bacharelado em Enfermagem - Campus Amilcar Ferreira Sobral da Universidade Federal do Piauí (UFPI), Floriano, Piauí.

ORCID: 0000-0003-0472-1852

\section{INTRODUÇÃO}

0 leite materno é a principal fonte de proteção e nutrição para a saúde do recém-nascido (RN), por possuir todos os componentes necessários para o crescimento e desenvolvimento saudável, além de favorecer o vínculo da díade mãe-filho ${ }^{(1)}$. Apesar desses benefícios, as prevalências de Aleitamento Materno Exclusivo (AME) e Aleitamento Materno Total das crianças estão abaixo das recomendações do Ministério da Saúde e Organização Mundial de Saúde $(\mathrm{OMS})^{(2)}$. Por esse motivo, é premente o desenvolvimento de estratégias de incentivo ao AME, consequentemente evitando o desmame precoce ${ }^{(3)}$.

Em todo o mundo, as taxas mais elevadas de aleitamento materno (AM) estão localizadas na África Subsaariana, no Sul da Ásia e em partes da América Latina. Em países de alta renda, a prevalência é inferior a $20,0 \%$. Já em países de baixa e média renda, 36,3 milhões $(63,0 \%)$ das crianças menores de seis meses não recebem $\mathrm{AME}^{(4)}$. No Brasil, identifica-se uma du- ração mediana de AME de 54,1 dias (1,8 meses) no conjunto das capitais brasileiras e Distrito Federal( ${ }^{(5)}$.

O Ministério da Saúde recomenda que o AM seja iniciado precocemente, ainda na primeira hora de vida do RN (chamada primeira hora de ouro) e mantido exclusivamente nos primeiros seis meses, quando passará a ser complementado até os dois anos ou mais ${ }^{(6)}$. No entanto, os dados identificados em pesquisas para tempo de AME mostram que os resultados encontram-se muito aquém do que é recomendado $^{(2)}$. Apesar de nas últimas três décadas, as prevalências dos indicadores de aleitamento materno no Brasil terem apresentado tendência ascendente, é preocupante a desaceleração identificada na mais recente Pesquisa Nacional de Saúde, realizada em 2013, que indicou queda no AME entre crianças de três a cinco meses de vida ${ }^{(7)}$.

A interrupção do AME ocorre por fatores diversos, geralmente associados a cultura, estilo de vida, baixa escolaridade da mulher, orientaçôes insuficientes dos profissionais de saúde durante o pré-natal e puerpério e, em alguns casos, por agravos de saúde do RN. Além disso, a técnica ineficaz da amamentação, que dificulta a sucção e esvaziamento mamário e pode levar a prejuízos na dinâmica da síntese de leite e problemas da mama, também é associada ao desmame precoce ${ }^{(1)}$.

Diante dos múltiplos fatores envolvidos na ocorrência do desmame precoce, reforça-se a necessidade de intensificar as estratégias já implementadas e desenvolver ações inovadoras de promoção, proteção e apoio ao aleitamento materno, abrangendo os variados setores da sociedade, no intuito de fomentar o crescimento da prevalência e duração do AME. Ademais, análises aprofundadas desse fenômeno também podem auxiliar na melhora dos indicadores ${ }^{(7)}$.

Frente a isso, esta pesquisa torna-se relevante por mostrar os intervenientes que podem levar ao desmame precoce no período neonatal. O conhecimento sobre os fatores de risco capazes de comprometer o aleitamento materno contribuirá para orientar intervenções e propiciar reflexôes sobre a qualidade da assistência no manejo 
do ciclo gravídico-puerperal na perspectiva de promoção e manutenção do AME. O estudo traz por objetivo analisar os fatores de risco para o desmame precoce no período neonatal destacados na literatura nacional e internacional. Desse modo, estabeleceu-se a seguinte questão norteadora da pesquisa: quais os fatores de risco para o desmame precoce no período neonatal?

\section{MÉTODO}

Trata-se de uma Revisão Integrativa da Literatura (RIL), em artigos científicos disponibilizados em periódicos nacionais e internacionais, armazenados nas bases de dados eletrônicas National Library of Medicine (PUBMED); Index to Nursing and Allied Health Literature (CINAHL); Literatura Latina Americana e do Caribe em Ciências da Saúde (LILACS), Índice Bibliográfico Español en Ciencias de la Salud (IBECS) e Base de Dados de Enfermagem (BDENF), via Biblioteca Virtual em Saúde (BVS); Literatura Inter- nacional em Ciências da Saúde (MEDILINE); WEB of SCIENCE e SCOPUS, nos idiomas português, inglês e espanhol. Foi utilizado o roteiro proposto na metodologia do Preferred Reporting Itens for Systematic Reviews and Meta-Analyses (PRISMA). Os dados foram coletados no mês de julho de 2019 por dois pesquisadores independentes, sem o estabelecimento de filtros temporais por se pretender uma análise integral da literatura sobre o tema.

Para elaboração do estudo foi adotada a estratégia PICo, em que o P corresponde ao Problema, I ao fenômeno de Interesse e Co ao Contexto de estudo ${ }^{(8)}$. Posteriormente, foram estabelecidos os descritores controlados e não controlados, selecionados a partir de consulta aos Descritores em Ciências da Saúde (DeCS), Medical Subject Headings (MeSH) e List of Headings do CINAHL Information Systems (Quadro1).

Foram estabelecidos como critérios de inclusão: estudos primários relacionados ao desmame precoce no período neona- tal, sem recorte temporal para seleção dos artigos. Excluiu-se: revisão narrativa de literatura/revisão tradicional, sistemática ou integrativa; notas, editoriais, relatórios, dissertações e teses; estudos que analisaram o desmame precoce no período neonatal associado a outros períodos (como menores de um ano) em que não seja possível delimitar especificamente o período neonatal; e estudos que não responderem ao questionamento da pesquisa.

A seleção dos estudos foi realizada, inicialmente, pela leitura dos títulos e resumos. Em seguida, os artigos selecionados foram lidos na íntegra, os dados extraídos foram agrupados em um formulário de coleta de dados adaptado ${ }^{(9)}$. Os dados obtidos mediante análise dos objetivos, resultados e conclusão de cada estudo foram agrupados por semelhança. A análise dos dados consistiu na codificação, categorização minuciosa e imparcial bem como na interpretação e síntese dos dados, com o objetivo de aumentar seu rigor a fim de entender o fenômeno estudado ${ }^{(10)}$.

\section{Quadro 1. Estratificação da pergunta de pesquisa seguindo a estratégia PICo. Floriano, PI, Brasil, 2020}

\begin{tabular}{|c|c|c|c|c|}
\hline PICo & COMPONENTES & DESCRITORES & DESCRITOR NÃO CONTROLADO & TIPOS \\
\hline$P$ & $\begin{array}{l}\text { Desmame } \\
\text { precoce }\end{array}$ & $\begin{array}{l}\text { Desmame } \\
\text { Weaning }\end{array}$ & $\begin{array}{l}\text { "Desmame precoce" } \\
\text { "Breastfeeding Weaning" } \\
\text { "Lactation Management" }\end{array}$ & $\begin{array}{c}\text { DeCS } \\
\text { MeSH } \\
\text { Títulos CINAHL }\end{array}$ \\
\hline I & Fatores de risco & $\begin{array}{l}\text { "Fatores de risco" } \\
\text { "Risk Factors" }\end{array}$ & $\begin{array}{c}\text { "Fator de Risco" } \\
\text { "Fatores de Risco Biológicos" } \\
\text { "Fatores de Risco Não Biológicos" } \\
\text { "Fatores de Riscos Biológicos" } \\
\text { "Fatores de Riscos Não } \\
\text { Biológicos" } \\
\text { "Factor, Risk" } \\
\text { "Factors, Risk" } \\
\text { "Risk Factor" }\end{array}$ & $\begin{array}{c}\text { DeCS } \\
\text { MeSH } \\
\text { Títulos CINAHL }\end{array}$ \\
\hline Co & Período neonatal & $\begin{array}{l}\text { "Recém-nascido" } \\
\text { "Infants, Newborn" }\end{array}$ & $\begin{array}{l}\text { "Criança Recém-Nascida" } \\
\text { "Crianças Recém Nascidas" } \\
\text { "Lactente Recém-Nascido" } \\
\text { "Lactentes Recém-Nascidos" } \\
\text { "Neonato" } \\
\text { "Neonatos" } \\
\text { "Newborn Infant" } \\
\text { "Newborn Infants" } \\
\text { "Newborns" } \\
\text { "Newborn" } \\
\text { "Neonate" } \\
\text { "Neonates" }\end{array}$ & $\begin{array}{c}\text { DeCS } \\
\text { MeSH } \\
\text { Títulos CINAHL }\end{array}$ \\
\hline
\end{tabular}




\section{artigo}

Lago, I.D.; Mesquita Silva, M.; Ferreira, M.S.; Rocha, K.N.S.; Rocha, R.C.; Bezerra, M.A.R.;

Fatores de risco para o desmame precoce no período neonatal: uma revisão integrativa da literatura

\section{RESULTADOS}

Foram identificados 1.673 artigos potencialmente relevantes para a revisão (MEDLINE via PUBMED $\mathrm{n}=336$; $\mathrm{LI}-$ $\mathrm{LACS} / \mathrm{BDENF} / \mathrm{IBECS}$ via BVS $\mathrm{n}=65$;
CINAHL $n=746$; WEB of SCIENCE $\mathrm{n}=62$; SCOPUS $\mathrm{n}=464$ ) dos quais 73 artigos foram selecionados para a leitura na íntegra, permanecendo um total de 12 artigos que preencheram os critérios de inclusão e foram selecionados para compor a

Figura 1. Fluxograma de seleção dos estudos segundo o Preferred Reporting Items for Systematic Reviews and Meta-Analyses (PRISMA). Floriano, PI, Brasil, 2020
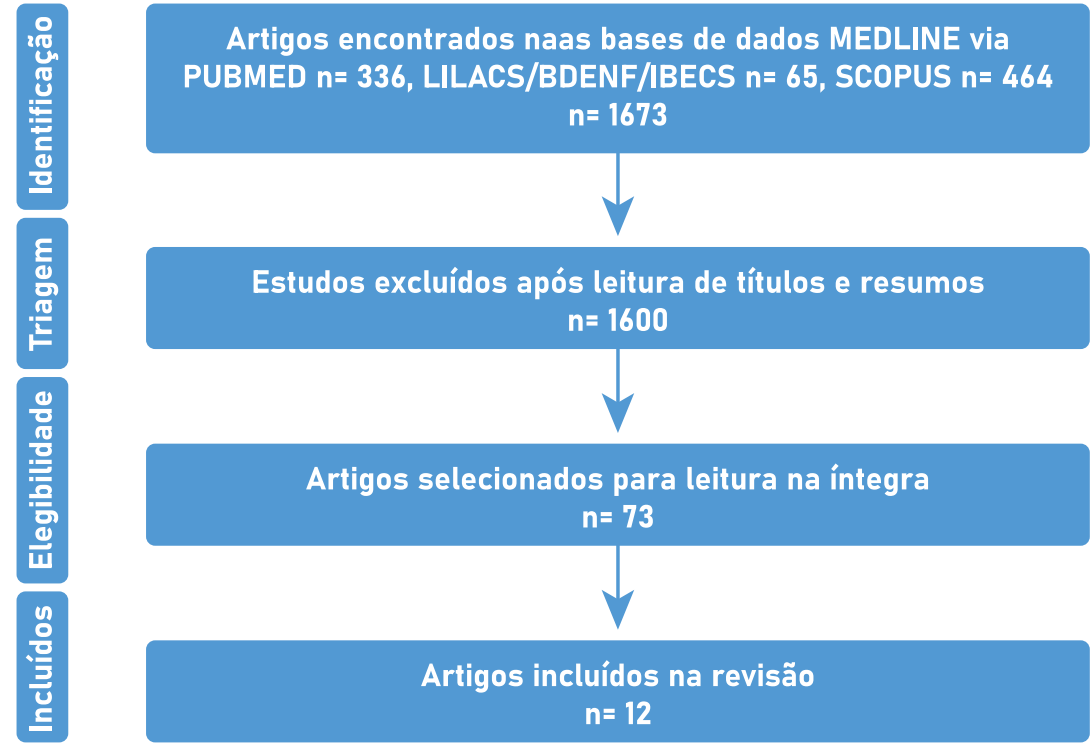

Artigos incluídos na revisão $n=12$

Quadro 2. Descrição dos artigos incluídos na RIL, segundo autor, objetivo e fatores de risco para o desmame precoce. Floriano, PI, Brasil, 2020s incluídos segundo autor, título, objetivo e resultado. Fortaleza, CE, Brasil, 2019

\begin{tabular}{|c|c|c|}
\hline $\begin{array}{l}\text { AUTOR / } \\
\text { CÓDIGO }\end{array}$ & OBJETIVO & FATORES DE RISCO PARA O DESMAME PRECOCE \\
\hline $\begin{array}{l}\text { Marques et } \\
\text { al..111) / A1 }\end{array}$ & $\begin{array}{l}\text { Descrever as práticas de amamentação } \\
\text { entre cri-anças de } 0 \text { a } 12 \text { meses de idade } \\
\text { em } 4 \text { pequenas cidades representativas } \\
\text { do Nordeste urbano do Brasil. }\end{array}$ & $\begin{array}{l}\text { Pré-estabelecimento da forma que pretende amamentar (não ex-clusiva); } \\
\text { acreditar que o bebê necessita de água, chá, suco e ou-tros alimentos; levar } \\
\text { chupeta e mamadeira para maternidade; ini-ciar outros leites, principalmen- } \\
\text { te em mamadeiras; receber alta da maternidade sem ter iniciado a amamen- } \\
\text { tação; considerar o ato de amamentar desagradável; choro do RN; volta ao } \\
\text { trabalho; acreditar que o bebê está sempre com fome. }\end{array}$ \\
\hline $\begin{array}{l}\text { Sheehan et al. } \\
\text { (12) / A2 }\end{array}$ & $\begin{array}{l}\text { Examinar os padrões de amamentação } \\
\text { entre os cinco estudos locais; Identificar } \\
\text { os fatores de risco para interrupção } \\
\text { precoce da amamenta-ção (ou seja, <4 } \\
\text { semanas após o parto) em Ontário. }\end{array}$ & $\begin{array}{l}\text { Alimentação com fórmula; inadequada oferta de leite; dificuldade nas } \\
\text { técnicas de amamentação; mamilos doloridos; falta de sistema universal de } \\
\text { apoio à amamentação; permanecer no hospital mais tempo do que } 48 \text { horas } \\
\text { após o nascimento do bebê; retorno ao trabalho ou escola; características } \\
\text { maternas (idade, paridade, ou-tros filhos, estado civil, renda, fonte de } \\
\text { renda, educação, status de emprego e prontidão para alta); necessidades } \\
\text { de aprendizado não atendidas no hospital; influência recebida favorável ao } \\
\text { uso de fórmula infantil; não concluir o ensino médio; não esperar confiar no } \\
\text { grupo ou centro de atendimento às mães; necessitar de uma ou mais visitas } \\
\text { maternas a um médico de familia; necessitar de cui-dados ou ajuda com a } \\
\text { amamentação, mas incapaz de obtê-la; não esperar contar com grupos de } \\
\text { mães ou centros de acolhimento. }\end{array}$ \\
\hline
\end{tabular}




\begin{tabular}{|c|c|c|}
\hline $\begin{array}{l}\text { Schwartz et } \\
\text { al. (13) / A3 }\end{array}$ & $\begin{array}{l}\text { Determinar os fatores demográficos, } \\
\text { compor-tamentais e clínicos as-sociados } \\
\text { à interrupção da amamentação nas pri- } \\
\text {-meiras } 12 \text { semanas pós-parto. }\end{array}$ & $\begin{array}{l}\text { Mastite; uso de bombas; dor nas mamas; uso de mamadeira; amamentar } \\
\text { seis ou menos vezes por dia; amamentar por menos de } 10 \text { minutos por ma- } \\
\text { mada; considerar o leite insuficiente; uso de mamadeiras; idade materna; } \\
\text { menor escolaridade; realizar suple-mentação alimentar com a fórmula in- } \\
\text { fantil. }\end{array}$ \\
\hline $\begin{array}{l}\text { Soares et al. } \\
\text { (14) / A4 }\end{array}$ & $\begin{array}{l}\text { Verificar a prática do uso de chupetas e } \\
\text { sua rela-ção com o desmame precoce em } \\
\text { crianças nascidas em um Hospital Amigo } \\
\text { da Criança. }\end{array}$ & Uso de chupeta; ser primogênito. \\
\hline $\begin{array}{l}\text { Taveras et al. } \\
\text { (15) / A5 }\end{array}$ & $\begin{array}{l}\text { Avaliar as associações entre a interrup- } \\
\text { ção da amamentação na } 2^{\mathrm{a}} \text { e } 12^{\mathrm{a}} \text { semana } \\
\text { pós-parto e apoio clínico, estado de saúde } \\
\text { física e mental materna, problemas no lo- } \\
\text { cal de trabalho e outros fatores passíveis } \\
\text { de inter-venção. }\end{array}$ & $\begin{array}{l}\text { Percepção de oferta insuficiente de leite; dor na mama; problemas com seus } \\
\text { recém-nascidos; problemas no local de trabalho ou es-cola; ser primípara } \\
\text { apresentar sintomas depressivos; falta de apoio do pai da criança; problemas } \\
\text { com a sucção ou pega do re-cém-nascido. }\end{array}$ \\
\hline $\begin{array}{l}\text { Venancio et } \\
\text { al. }{ }^{(16)} / \mathrm{A6}\end{array}$ & $\begin{array}{l}\text { Identificar fatores associ-ados à introdu- } \\
\text { ção de líquidos, exceto leite ma-terno, no } \\
\text { primeiro dia em casa após alta do hospi- } \\
\text {-tal. }\end{array}$ & Introdução de água, chá e outros leites; parto cesáreo. \\
\hline $\begin{array}{l}\text { Gerd et al. }{ }^{(17)} \\
\quad \text { / A7 }\end{array}$ & $\begin{array}{l}\text { Avaliar os fatores associ-ados à desconti- } \\
\text { nuação da amamentação durante as pri- } \\
\text { meiras } 4 \text { semanas. }\end{array}$ & $\begin{array}{l}\text { Introdução de misturas e fórmula infantil; dificuldade para iniciar o aleita- } \\
\text { mento materno; problemas no processo de amamentação; tabagismo ma- } \\
\text { terno e paterno; baixo peso ao nascimento; técnica de sucção do bebê ina- } \\
\text { dequada; percepção de baixo ganho de peso; problema nos mamilos; pega e } \\
\text { suç̧ão inadequada; baixa frequência de mamadas; prematuridade. }\end{array}$ \\
\hline $\begin{array}{l}\text { Rius et al. }{ }^{(18)} \\
\quad / \text { A8 }\end{array}$ & $\begin{array}{l}\text { Analisar possiveis fatores associados ao } \\
\text { abandono do AM. }\end{array}$ & $\begin{array}{l}\text { Introdução de fórmula artificial; perda de apoio à amamentação após a alta } \\
\text { da maternidade; sensação de produção de leite insufi-ciente; fumar durante } \\
\text { a gravidez; ter alimentado a criança anterior com leite artificial; baixa expec- } \\
\text { tativa materna em amamentar; es-tresse emocional, pessoal e econômico } \\
\text { dessas situações. }\end{array}$ \\
\hline $\begin{array}{l}\text { Lindau et al. } \\
\text { (19) / A9 }\end{array}$ & $\begin{array}{l}\text { Investigar os fatores de risco para a ama- } \\
\text { menta-ção exclusiva. }\end{array}$ & $\begin{array}{l}\text { Uso de chupetas; cesárea planejada; sofrimento psíquico como: episódios de } \\
\text { depressão, insônia, angústia e percepção do nasci-mento como evento trau- } \\
\text { mático; não frequentar aulas pré-natais; apresentar estresse físico e mental }\end{array}$ \\
\hline $\begin{array}{l}\text { Amaral et al. } \\
\text { (20) / A10 }\end{array}$ & $\begin{array}{l}\text { Identificar os fatores que podem in uen- } \\
\text { ciar as nutrizes na interrupção do alei- } \\
\text { tamento materno exclusivo durante os } \\
\text { pri-meiros seis meses de vida do lactente. }\end{array}$ & $\begin{array}{l}\text { Crença de produção de leite insuficiente ou de leite fraco nos pri-meiros dias } \\
\text { do puerpério; insegurança materna; dificuldade de pega; intercorrências ma- } \\
\text { márias relacionadas a pega ou posicio-namento inadequados da criança ao } \\
\text { mamar; dor durante o alei-tamento; realizar aleitamento materno predo- } \\
\text { minante; apresentar intercorrências com o neonato; uso de bicos artificiais } \\
\text { ou mama-deira; mitos e crenças relacionados com o ato de amamentar que } \\
\text { in uenciam diretamente na introdução precoce de outros líquidos, como os } \\
\text { chás e a água; falta de apoio dos familiares e dos profis-sionais de saúde. }\end{array}$ \\
\hline $\begin{array}{l}\text { Karall et al. (21) } \\
\quad \text { / A11 }\end{array}$ & $\begin{array}{l}\text { Entender melhor os fato-res que influen- } \\
\text { ciam o desmame em nossa re-gião. }\end{array}$ & $\begin{array}{l}\text { Alimentação suplementar; baixa idade materna; baixo nível educa-cional; in- } \\
\text { suficiência de leite percebida; baixa autoeficácia na amamentação. }\end{array}$ \\
\hline $\begin{array}{l}\text { Vila-Candel et } \\
\text { al. }{ }^{(22)} / \text { A12 }\end{array}$ & $\begin{array}{l}\text { Determinar a prevalência de aleitamento } \\
\text { materno exclusivo aos } 3 \text { meses pós-par- } \\
\text { to; Conhecer as causas que contribuem } \\
\text { para o abandono precoce. }\end{array}$ & $\begin{array}{l}\text { Motivos relacionados à glândula mamária (mamilo umbilicado; mastite; fis- } \\
\text { suras no mamilo; hipogalactia); Motivos relacionados ao recém-nascido (do- } \\
\text { ença neonatal, icterícia, internação, baixo peso e prematuridade); perda de } \\
\text { assistência ao aleitamento materno; desejo da puérpera em utilizar fórmula } \\
\text { artificial; ganho de peso do recém-nascido menor que o recomendado; fato- } \\
\text { res trabalhistas. }\end{array}$ \\
\hline
\end{tabular}

significativa entre a introdução de fórmula artificial/suplementação alimentar e o desmame precoce, conforme se verifica em A1, A2, A3, A6, A7, A8, e A11. Esse fator de risco, quando realizado no período neona- tal, traz impactos negativos para manutenção do AM, destacando-se entre os demais identificados, em virtude desses alimentos possuírem alto peso calórico e difícil digestão. Como consequência, o RN apresenta maior saciedade pela dificuldade de seu sistema gastrointestinal, ainda imaturo, em metabolizar essas partículas estranhas ${ }^{(24)}$.

Além disso, em geral, esses alimentos são disponibilizados em mamadeiras, o 
que provoca desestímulo em sugar a mama e prejudica a estimulação neuromotora do complexo crânio-face do RN. Contribui, ainda, para o abuso alimentar, já que as mães muitas vezes esperam que seus $\mathrm{RN}$ consumam todo o conteúdo disponibilizado. Esses fatores favorecem, assim, a diminuição da necessidade do RN em ser aleitado à mama, colaborando para o surgimento de alguns problemas de amamentação, como a diminuição da produção de leite e a ocorrência de fissuras mamilares ${ }^{(25,26)}$.

Também destacaram-se como intervenientes alterações anatômicas, fisiológicas ou patológicas, tais como: dor, mastite, mamilo umbilicado, fissuras no mamilo e hipogalactia, como evidenciado em A2, A3, A5, A7, A10, e A12. Esses problemas mamários estão dentre as principais causas que levam ao desmame precoce e tendem a surgir nas primeiras semanas de puerpério. Eles desencadeiam desconforto e dificuldade em amamentar, pois, quando a nutriz sente dor, estresse ou medo, seu organismo não libera ocitocina, hormônio fundamental para ejeção do leite ${ }^{(1,3)}$.

Evidenciou-se, ainda, fatores de risco relacionados à técnica de amamentação em A2, A3, A5, A7 e A10, a saber: pega inadequada, mamadas de curta duração e com tempo determinado e troca frequente da mama. Essas práticas inadequadas impossibilitam o consumo do leite posterior, que propicia maior saciedade ao $\mathrm{RN}$ e, por conseguinte, potencializam os problemas mamários, já que podem levar ao traumatismo da mama, além de contribuir para tornar a técnica de amamentação ineficaz pela presença de dor e desconforto associados ao ato de amamentar ${ }^{(27)}$.

Um outro problema comum à lactação é a percepção / sensação de produção de leite insuficiente ou leite fraco, levando a mãe a acreditar que o RN chora constantemente em decorrência da fome, conforme foi verificado em A1, A3, A5, A6, A10, A11 e A12. As dificuldades no aleitamento materno favorecem a diminuição da produção de leite que através do mecanismo de feedback negativo, pelo não esvaziamento adequado das mamas, torna a produção de leite cada vez menor, até cessar completa- mente $^{(28)}$. É fundamental esclarecer ainda durante o pré-natal que faz parte do comportamento natural dos recém-nascidos mamar com frequência, o que é interpretado erroneamente como sinal de fome da criança, leite fraco ou insuficiente, predispondo à complementação com fórmulas e, consequentemente, ao desmame ${ }^{(25)}$.

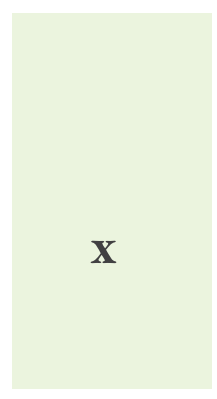

Intercorrências com o neonato, entre elas prematuridade, que se relaciona diretamente a baixo peso ao nascer, doença neonatal, icterícia e internação, também estão entre os principais achados que influem negativamente no AME, e foram relatadas em A1, A2, A5, A7, e A10. Em especial, devido à imaturidade fisiológica, neurológica, hipotonia muscular e hiporreatividade aos estímulos do meio ambiente ${ }^{(1)}$, a prematuridade apresenta risco associado ao desmame precoce relacionado, de modo significativo, ao reflexo de sucção débil, fraco ou ausente ${ }^{(29)}$. Apesar das dificuldades interpostas, estratégias que permitam a oferta do leite materno para RN prematuros devem ser implementadas, entre elas a administração via sonda orogástrica por gavagem, por meio de gastróclise, bem como através do uso do copinho ${ }^{(30)}$.

O uso de utensílios culturais como chupetas e mamadeiras, identificados em A1, A3 A4, A9 e A10, também mostra-se associado ao desmame, pois os lactentes que os utilizam tendem a deixar precocemente a mama, em virtude de facilitarem a confusão de bicos ${ }^{(2)}$. Ademais, o uso da mamadeira favorece o surgimento de alterações neuromusculares, oclusivas, de deglutição, mastigação, e aumentam a probabilidade do desenvolvimento de cáries dentárias, além de estar associado a maior risco de doenças infecciosas ${ }^{(26)}$.

A retomada às atividades cotidianas, especialmente o retorno às atividades laborais e/ou escolares, como relatado em A1, A2, A5 e A12, também se apresentou como fator de risco relevante para o desmame precoce. A reinserção nessas atividades desencadeia sobrecarga materna, pela necessidade de conciliação de múltiplos papéis ${ }^{(3)}$, e impõe uma rotina diária mais atarefada que acaba predispondo o uso de bicos artificiais, como chupeta e mamadeira, por permitir a ingestão do alimento mais rapidamente e pela liberdade para realização de outras atividades, já que outra pessoa pode oferecê-la ao RN. Assim, o uso desses dispositivos, conforme anteriormente referido, contribui para o desmame precoce ${ }^{(26)}$.

Os problemas de ordem psicológica, como estresse, angustia, insônia, percepção do nascimento da criança como um evento traumático e depressão, evidenciados em A2, A5, A8 e A9, também são frequentes nas primeiras semanas de puerpério e constituem fatores de risco relevantes para o desmame precoce. Existe uma relação inversa entre esses agravos, em es- 
pecial depressão pós-parto, e aleitamento materno, em virtude de uma menor probabilidade de ter uma experiência positiva, o que pode levar ao desmame precoce ${ }^{(31)}$. A identificação precoce de problemas de saúde mental materna durante o período perinatal é essencial para promoção do bem-estar emocional das mães e prevenir dificuldades para amamentar ${ }^{(32)}$.

\section{CONCLUSÃO}

A análise dos artigos evidenciou a complexidade da prática do AM e o caráter multifatorial do desmame precoce. Verificou-se a presença de diferentes fatores em um mesmo contexto de ocorrência do desmame precoce, seja envolvendo aspectos anatomofisiológicos ou culturais, psicológicos, econômicos, sociais que, mutuamente, são capazes de potencializar os riscos e dificultar a implementação e manutenção do AM no período neonatal e, como consequência, em fases posteriores.

$\mathrm{O}$ quantitativo significativo de fatores de risco associados à interrupção precoce do AM já nas primeiras horas e dias de vida do RN permite indicar a importância da análise e identificação antecipada de possíveis intervenientes que podem ser rastreados ainda na gestação, através da consulta pré-natal, ou no Alojamento Conjunto, na perspectiva de preveni-los ou manejá-los. Reforça-se, ainda, a im- portância de se investir em estratégias que reduzam os fatores de risco, considerando os diferentes contextos nos quais as mães e RN estão inseridos. Assim, necessita-se de uma abordagem ampliada, individualizada e multiprofissional.

\section{AGRADECIMENTOS}

Agradecemos ao CNPq, edital Chamada MCTIC/CNPq No 28/2018, processo 420768/2018-1, pelo apoio e financiamento do macroprojeto: "Efetividade de uma intervenção educativa por telefone na promoção do aleitamento materno no período neonatal", ao qual o presente estudo encontra-se vinculado.

\section{REFERÊNCIAS}

1. Urbanetto PDG, Costa AR, Gomes GC, Nobre CMG, Xavier DM, Jung BC. Facilidades e dificuldades encontradas pelas puérperas para amamentar/Facilites and difficulties found by mothers to beastfeed. Revista de Pesquisa: Cuidado é Fundamental Online [serial on Internet]. 2018 [cited 2019 nov 4]; 10 (2): 399-405. Available from: http://dx.doi.org/10.9789/2175-5361.2018. v10i2.399-405

2. Santos EMD, Silva LSD, Rodrigues BFDS, Amorim TMAXD, Silva CSD, Borba JMC, et al. Avaliação do aleitamento materno em crianças até dois anos assistidas na atenção básica do Recife, Pernambuco, Brasil. Ciência \& Saúde Coletiva [serial on Internet]. 2019 [cited 2020 fev 18]; 24: 1211-1222. Available from: https://doi.org/10.1590/1413-81232018243.126120171

3. Souza DR, Diógenes SM, Andrade JSO, Oliveira PCP. Aleitamento materno e os motivos do desmame precoce no município de Porto Velho/RO. Revista Eletrônica Acervo Saúde [serial on Internet]. 2019 [cited 2020 mar 24]; (31): e1087-e1087. Available from: https://doi.org/10.25248/reas.e1087.2019

4. Victora CG, Bahl R, Barros AJD, França GVA, Horton S, Krasevec $\mathrm{J}$ et al. Breastfeeding in the 21st century: epidemiology, mechanisms, and lifelong effect. Lancet [serial on Internet]. 2016 [cited 2020 fev 18]; 387(10017): 475-90. Available from: https://doi. org/10.1016/S0140-6736(15)01024-7

5. Ministério da Saúde (BR). Secretaria de Atenção à Saúde. Departamento de Ações Programáticas e Estratégicas. II Pesquisa de Prevalência de Aleitamento Materno nas Capitais Brasileiras e Distrito Federal [Internet]. Brasília: Ministério da Saúde; 2009 [citado 2020 mar 6]. 180 p. Available from: http://bvsms.saude. gov.br/bvs/publicacoes/pesquisa_prevalencia_aleitamento_ materno.pdf

6. Ministério da Saúde (BR). Secretaria de Atenção à Saúde. Departamento de Ações Programáticas Estratégicas. Política nacional de atenção integral à saúde da criança: orientações para implementação [Internet]. Brasília: Ministério da Saúde; 2018 [citado 2020 mar 6]. 180 p. Available from: https://portaldeboaspraticas.iff.fiocruz.br/biblioteca/pnaisc/

7. Boccolini CS, Boccolin, PDMM, Monteiro FR, Venâncio SI, Giugliani ERJ. Tendência de indicadores do aleitamento materno no Brasil em três décadas. Revista de Saúde Pública [serial on Internet]. 2017 [citado 2020 mar 6]; 51: 108. Available from: https:// doi.org/10.11606/S1518-8787.2017051000029

8. Karino ME, Felli VEA. Enfermagem baseada em evidências: avanços e inovações em revisões sistemáticas. Ciência, Cuidado e Saúde [serial on Internet]. 2012 [cited 2019 jun 17]; 11: 011015. Available from: https://doi.org/10.4025/cienccuidsaude. v11i5.17048

9. Ursi ES, Galvão CM. Perioperative prevention of skin injury: an integrative literature review. Revista Latino-Americana de Enfermagem [serial on Internet]. 2006 [cited 2019 jun 17]; 14 (1): 124-131. Available from: https://www.scielo.br/pdf/rlae/v14n1/ v14n1a17

10. Whittemore $R$, Knafl K. The integrative review: updated methodology. Journal of advanced nursing [serial on Internet]. 2005 [cited 2019 jan 07]; 52(5), 546-553. Available from: https:// doi.org/10.1111/j.1365-2648.2005.03621.x

11. Marques NM, Lira PI, Lima MC, Silva NL, Batista Filho M, Huttly SR, et al. Breastfeeding and early weaning practices in northeast Brazil: a longitudinal study. Pediatrics [serial on Internet]. 2001 [cited 2019 jul 03]; 108 (4): e66-e66. Available from: https://doi.org/10.1542/peds.108.4.e66

12. Sheehan D, Krueger P, Watt S, Sword W, Bridle B. The Ontario mother and infant survey: breastfeeding outcomes. Journal of human lactation [serial on Internet]. 2001 [cited 2019 jul 03]; 17 (3): 211-219. Available from: https://doi.org/10.1177\% 2F089033440101700304 


\section{artigo}

Lago, I.D.; Mesquita Silva, M.; Ferreira, M.S.; Rocha, K.N.S.; Rocha, R.C.; Bezerra, M.A.R.

Fatores de risco para o desmame precoce no período neonatal: uma revisão integrativa da literatura

\section{REFERÊNCIAS}

13. Schwartz K, D'Arcy HJ, Gillespie B, Bobo J, Longeway M, Foxman B. Factors associated with weaning in the first 3 months postpartum. Journal of Family Practice [serial on Internet]. 2002 [cited 2019 jul 26]; 51 (5): 439-444. Available from: https://europepmc.org/article/med/12019051

14. Soares MEM, Giugliani ERJ, Braun ML, Salgado ACN, Oliveira $A P$, et al. Uso de chupeta e sua relação com o desmame precoce em população de crianças nascidas em Hospital Amigo da Criança. J Pediatr (Rio J) [serial on Internet]. 2003 [cited 2019 jul 26]; 79 (4): 309-16. Available from: http://dx.doi.org/10.1590/ S0021-75572003000400008

15. Taveras EM, Capra AM, Braveman PA, Jensvold NG, Escobar GJ, Lieu TA. Clinician support and psychosocial risk factors associated with breastfeeding discontinuation. Pediatrics [serial on Internet]. 2003 [cited 2019 jul 16]; 112 (1): 108-115. Available from: https://doi.org/10.1542/peds.112.1.108

16. Venancio SI, Saldiva SR, Mondini L, Levy RB, Escuder MML. Early interruption of exclusive breastfeeding and associated factors, state of São Paulo, Brazil. J Hum Lact [serial on Internet]. 2008 [cited 2019 jul 11]; 24 (2):168-174. Available from: https:// doi.org/10.1177\%2F0890334408316073

17. Gerd AT, Bergman S, Dahlgren J, Roswall J, Alm B. Factors associated with discontinuation of breastfeeding before 1 month of age. Acta Paediatrica [serial on Internet]. 2012 [cited 2019 jul 11]; 101 (1): 55-60. Available from: https://doi.org/10.1111/ j.1651-2227.2011.02405.x

18. Rius JM, Ortuño J, Rivas C, Maravall M, Calzado MA, López $A$, et al. Factores asociados al abandono precoz de la lactancia materna en una región del este de España. Anales de pediatria [serial on Internet]. 2014 [cited 2019 jul 26]; 80 (1): 6-15. Available from: https://doi.org/10.1016/j.anpedi.2013.05.011

19. Lindau JF, Mastroeni S, Gaddini A, Di Lallo D, Nastro PF, Patanè $\mathrm{M}$, et al. Determinants of exclusive breastfeeding cessation: identifying an "at risk population" for special support. European journal of pediatrics [serial on Internet]. 2015 [cited 2019 jul 26]; 174 (4): 533-540. Available from: http://dx.doi.org/10.1007/ s00431-014-2466-4

20. Amaral LJX, Sales SDS, Carvalho DPDSRP, Cruz GKP, Azevedo ICD, Ferreira Júnior MA. Fatores que influenciam na interrupção do aleitamento materno exclusivo em nutrizes. Revista gaúcha de enfermagem [serial on Internet]. 2015 [cited 2019 jul 26]; 36 (SPE): 127-134. Available from: https://doi.org/10.1590/19831447.2015.esp.56676

21. Karall D, Ndayisaba JP, Heichlinger A, Kiechl-Kohlendorfer $\mathrm{U}$, Stojakovic S, Leitner $\mathrm{H}$, et al. Breast-feeding Duration: Early Weaning-Do We Sufficiently Consider the Risk Factors? Journal of pediatric gastroenterology and nutrition [serial on Internet]. 2015 [cited 2019 jul 11]; 61 (5): 577-582. Available from: 0.1097/MPG.0000000000000873

22. Vila-Candel $R$, Soriano-Vidal FJ, Murillo-Llorente $M$, Pérez-Bermejo M, Castro-Sánchez E. Mantenimiento de la lactancia materna exclusiva a los 3 meses posparto: experiencia en un departamento de salud de la Comunidad Valenciana. Atención
Primaria [serial on Internet]. 2019 [cited 2019 jul 13]; 51 (2): 91 98. Available from: https://doi.org/10.1016/j.aprim.2017.09.002

23. DeCS (Descritores de Ciências em Saúde). Site. 2020. Disponivel em: http://decs.bvs.br/cgibin/wxis1660.exe/decsserver/ Acesso em: 15 de agosto de 2020.

24. Hockenberry MJ, Wilson D. Wong: Fundamento de Enfermagem Pediátrica. 9 ed. Rio de Janeiro: Elsevier, 2014.

25. Sousa ELO, Melo LGNS, Medeiros DMF. Práticas de complementação ao leite materno: concepções de puérperas sobre aleitamento materno e uso de fórmula infantil. Revista Brasileira de Educação e Saúde [serial on Internet]. 2019 [cited 2020 jun 21]; 9 (2): 76-84. Available from: https://doi.org/10.18378/ rebes.v9i2.6149

26. Bezerra VM, Magalhães EIDS, Pereira IN, Gomes AT, Pereira NM, Rocha DS. Prevalência e fatores determinantes do uso de chupetas e mamadeiras: um estudo no sudoeste baiano. Revista Brasileira de Saúde Materno Infantil [serial on Internet]. 2019 [cited 2020 jun 21]; 19 (2): 311-321. Available from: http://dx. doi.org/10.1590/1806-93042019000200004

27. Yilak G, Gebretsadik W, Tadesse H, Debalkie M, Bante A. Prevalence of ineffective breastfeeding technique and associated factors among lactating mothers attending public health facilities of South Ari district, Southern Ethiopia. PloS one [serial on Internet]. 2020 [cited 2020 jun 21];15(2), e0228863. Available from: https://doi.org/10.1371/journal.pone.0228863

28. Rodrigo R, Rodrigo A, Liyanage N, Hatahagoda W, Hewavitharana U. Maternal Perception of Adequacy of Mother's Milk Among Mothers Giving Birth at a Teaching Hospital in Sri Lanka. Journal of Human Lactation [serial on Internet]. 2019 [cited 2020 jul 01]; 35 (1): 171-180. Available from: https://doi. org/10.1177\%2F0890334418773304

27. Mattsson E, Funkquist EL, Wickström M, Nyqvist KH, Volgsten $\mathrm{H}$. Healthy late preterm infants and supplementary artificial milk feeds: Effects on breast feeding and associated clinical parameters. Midwifery [serial on Internet]. 2015 [cited 2020 jul 01]; 31 (4): 426-431. Available from: https://doi.org/10.1016/j. midw.2014.12.004

30. Nascimento J, Santos IMMD, Silva LJD. Cuidados com recém-nascidos alimentados por sonda gástrica: conceitos e práticas. Texto \& Contexto-Enfermagem [serial on Internet]. 2019 [cited 2020 jul 01]; 28. Available from: http://dx.doi. org/10.1590/1980-265x-tce-2017-0242

31. Webber E, Benedict J. Postpartum depression: a multi-disciplinary approach to screening, management and breastfeeding support. Archives of psychiatric nursing [serial on Internet]. 2019 [cited 2020 jun 21]; 33 (3): 284-289. Available from: https://doi. org/10.1016/j.apnu.2019.01.008

32. Coo S, García MI, Mira A, Valdés V. The Role of Perinatal Anxiety and Depression in Breastfeeding Practices. Breastfeeding Medicine. [serial on Internet]. 2020; [cited 2020 ago 15]15 (8): 495-500. Available from: https://doi.org/10.1089/ bfm.2020.0091 